\title{
Mathis-Moser, Ursula \& MArie Carrière (eds.). 2019. Écrire au-delà de la fin des temps? Les littératures au Canada et au Québec/Writing beyond the end of times? The literatures of Canada and Quebec. Insbruck, Insbruck university press, col. "canadiana oenipontana" 14, ISBN: 978-1-77212-507-8, 280 pp.
}

Dégradation environnementale, violence mondiale, croissance des divisions sociales, crise du multiculturalisme..., l'évolution de notre monde occidental semble approcher un point zéro apocalyptique qui trouve son reflet dans la littérature.

Les éditrices Ursula Mathis-Moser et Marie Carrière ont publié, à la suite du colloque bilingue (français-anglais) Crisis and Beyond: The Literatures of Canada and Quebec/Écriture de la crise: dans la tourmente et au-delà. Les littératures au Canada et au Québec, célébré à l'Université d'Innsbruck en automne 2015, ce volume d'exploration collective (innsbruck university press, col. canadiana oenipontana 14, 2017) qui s'interroge sur le rôle de la littérature face au sentiment de crise dominant dans notre société actuelle, après le tournant du millénaire. L'ouvrage convainc le lecteur de la pertinence des interventions des écrivains canadien-ne-s et québécois-e-s à cet égard. En effet, la réflexion part d'une approche volontairement transnationale, transfrontalière, interdisciplinaire, postcoloniale et féministe et se pose - et nous pose - la question fondamentale sur comment l'écriture de la mobilisation, de l'engagement ou de la transgression pourrait contrer la crise et inspirer à l'humanité un nouvel espoir en ouvrant la voie à la transformation sociale.

Les articles qui conforment le volume sont organisés autour de cinq axes principaux: l'apocalypse et la dystopie dans les manifestations littéraires du XXIe siècle, l'empathie et les nouveaux cadres éthiques de l'amour et du soin qui peuvent surmonter la répression et l'aliénation sociétale, les stratégies créatives et esthétiques qui peuvent aider à affronter ou surmonter la crise, la réflexion critique sur "la mémoire", "l'histoire" et "l'oubli" selon les concepts bien connus de Paul Ricœur, et finalement les conflits culturels à l'intérieur de la société canadienne et québécoise. 
Le premier apport fondamental de ce volume réside d'abord dans la diversité des réponses proposées par les chercheur.e.s participant dans ce volume collectif. Provenant de différents pays et d'horizons universitaires divers, David Boucher, Nicole Côté, Piet Defraeye, Nicoletta Dolce, Danielle Dumontet, Ana María Fraile-Marcos, Andrea Krotthammer, Marion Kühn, Hans-Jürgen Lüsebrink, Carmen Mata Barreiro, Dunja M. Mohr, Émilie Notard, Daniel Poitras, Véronique Porra, Srilata Ravi et Marion Christina Rohrleitner ouvrent une réflexion plurielle, en anglais ou en français selon leur choix, sur les enjeux de l'écriture au-delà du sentiment de crise. En effet, les réponses de ces auteurs face à une société individualiste, nihiliste, capitaliste, néolibérale, aliénée et en pleine crise existentielle sont multiples. Dans l'utopie/dystopie, la littérature peut (re)trouver l'espoir, à la fois lucide et désenchanté (Dolce), dans le fait de s'investir dans la narration même (Mohr), où la littérature exerce son pouvoir de témoigner de "ce monde courant à sa perte" (Côté). L'écriture peut également nous mettre face au miroir cauchemardesque du memento mori littéraire (Boucher). Des réponses peuvent être trouvées dans des nouveaux cadres éthiques proposant un éveil de conscience politique de l'amour au-delà du romantisme (Fraile-Marcos). Dans un monde où la littérature devient espace fondamentale de réflexion, les écrivains sont des référents nécessaires d'intégrité intellectuelle, illustration vivante de résistance, d'empathie et de responsabilité tournée vers le futur (Mata Barreiro). La quête de sens contre l'inhumanité devient à son tour un plaidoyer pour la vie malgré tout, malgré l'inquiétude qui peut persister (Notard). Les arts scéniques comme le théâtre proposent, quant à eux, un langage imaginatif ainsi que le jeu comme puissante stratégie de résistance face à la crise (Defraeye). Le corps est représenté dans le jeune cinéma québécois comme l'écran visible, l'interface symbolique entre un malaise intérieur indéfini et un monde étranger (Porra). La crise se présente également sous forme d'oubli ravageur de la mémoire collective, ou de mémoire manipulée, proposant la fiction comme enquête face à l'impossibilité d'aller au fond de la "vérité" (Kühn). Les héritiers prennent ainsi la parole dans la narration d'une post-mémoire qui montre que le passé habite le présent (Dumontet). Ce présent omniprésent qui met en crise l'historicité évoque, sur le plan symbolique, la situation du Québec en Amérique (Poitras) et repense le multiculturalisme et l'interculturalisme dans le contexte québécois et canadien (Lüsebrink). En particulier, la littérature permet de s'interroger sur les relations de pouvoir et les bonnes intentions, noyées dans la langue eurocentrique, dans la représentation des peuples autochtones (Ravi). Finalement, la littérature latina transnationale et multilingue va au-delà de la littérature d'exil, montrant la puissance de la latinidad dans l'identité américaine, tant aux Etats-Unis qu'au Canada (Rohrleitner).

En second lieu, il convient de souligner la richesse des textes et des écrivains étudiés dans chacun des articles. De Margaret Atwood à Nelly Arcan en passant par Louise Dupré, Dionne Brand, Simone Chaput, Gérard Bouchard, Jean-François Caron, Mario Brassard, Wajdi Mouawad, Xavier Dolan, Anne Émond, Abla Farhoud, Nicole Brossard, Monique 
La Rue, Carl Leblanc, Jacques Poulin, Alfonso Quijada Urías, Nela Rio, Chloé Robichaud, Carmen Rodríguez, Jocelyne Saucier, Larry Tremblay, Michel Marc Bouchard ou Catherine Mavrikakis, nombreux sont les auteurs, tant anglophones que francophones abordés dans la complexité de leurs œuvres littéraires, théâtrales ou cinématographiques dont l'analyse des collaborateurs ouvre de nouvelles pistes d'analyse. La profondeur, l'originalité et l'ouverture transdisciplinaire de ces nouvelles pistes explorées est le troisième apport remarquable de cet ouvrage.

Tel que l'expose ce volume collectif, le rôle de la littérature est donc pluriel face à la crise: elle confronte le lecteur face à des 'logiques nouvelles', mais elle l'encourage également à aller de l'avant. La littérature nourrit l'espoir et propose de nouvelles stratégies de résistance: l'éthique de l'empathie et du care, l'importance de la mémoire et du témoignage, la politique de l'amour et finalement l'utilisation d'un langage imaginatif qui peut devenir subversif grâce à l'ironie, l'humour et la dérision.

Dans ce monde contemporain de l'Après 11 septembre, la littérature devient un outil extraordinaire de quête de sens, de redécouverte de la beauté, et les études littéraires seraient par conséquent une vraie "science de la vie" facilitant la réflexion sur un "savoir-vivre" critique, si nécessaire face aux défis de notre monde actuel.

Beatriz Calvo Martín Université libre de Bruxelles 
